\title{
DETECCCIÓN TEMPRANA DE LA DISGRAFÍA DESDE EL EJERCICIO PROFESIONAL DOCENTE
}

\section{THE EARLY DETECTION OF DYSGRAPHIA FROM THE TEACHING PROFESSION}

\author{
Eddy Suyen Vargas Rivera ${ }^{1}$
}

Recibido: 2021-10-04 / Revisado: 2021-11-10 / Aceptado: 2021-11-25 / Publicado: 2022-01-01

Forma sugerida de citar: Vargas-Rivera, E. S. (2022). Detección temprana de la disgrafía desde el ejercicio profesional docente. Retos de la Ciencia. 6(12). 13-24. https://doi.org/10.53877/rc.6.12.20220101.02

\section{RESUMEN}

En este trabajo se analiza la importancia de detectar de manera temprana la Disgrafia en el ejercicio profesional docente de los primeros años de escolaridad, lo cual, permite intervenir oportunamente y evitar el progreso de esta problemática que en el fututo podría convertirse en un Trastorno Específico de Aprendizaje. Se trata de una investigación cualitativa que recurrió al método de la investigación acción desde la práctica de 102 profesores que se encuentran cursando la Carrera de Educación Básica de la Universidad Tecnológica Indoamérica. Los resultados demuestran que el mayor porcentaje de docentes investigadores realizan la detección de la Disgrafía en los primeros años de escolaridad recurriendo a métodos observacionales aplicados en las actividades escolares cotidianas. Se concluye que la detección de la disgrafía en el contexto investigado es limitada, por lo que aporta escasamente en la solución de la referida dificultad de aprendizaje.

Palabras clave: aprendizaje, detección de disgrafía, dificultad de aprendizaje, disgrafía

\section{ABSTRACT}

This work analyzes the importance of early detection of Dysgraphia in the professional teaching practice of the first years of schooling, which allows timely intervention and prevent the progress of this problem that in the future could become a Specific Disorder of Learning. It is qualitative research that used the action research method from the practice of 102 professors who are studying the Basic Education Career of

\footnotetext{
${ }^{1}$ Doctora en Psicóloga Infantil y Psicorehabilitación. Profesora a tiempo completo en la Universidad Tecnológica Indoamérica. Ecuador. E-mail: eddyvargas@indoamerica.edu.ec / ORCID: https://orcid.org/0000-0002-2069-5730
} 
the Indoamérica Technological University. The results show that the highest percentage of research teachers carry out the detection of Dysgraphia in the first years of schooling by resorting to observational methods applied in daily school activities. It is concluded that the detection of dysgraphia in the investigated context is limited, therefore it contributes little to the solution of the referred learning difficulty.

Keywords: learning, dysgraphia detection, learning difficulty, dysgraphia

\section{INTRODUCCIÓN}

La formación de profesionales de la educación debe ser integral en cuyo proceso el aprendizaje de estrategias para detectar e intervenir en dificultades de aprendizaje juega un papel esencial. Cabe señalar que la escritura se desarrolla a partir de un amplio proceso que se va consolidando progresivamente en los distintos niveles de Educación Básica es ahí donde justamente se requiere de una apropiada y oportuna intervención del docente para identificar dificultades de escritura, lo cual, se apoya en el criterio de la OMS citado en Gonzáles (2020) " [...] Se trata de mantener el estado de salud reduciendo los factores de riesgo antes de que produzcan más daños y si ya existiera un trastorno instalado, impedir su progresión." (p.12). Este enunciado insta la necesidad de detectar tempranamente las dificultades de la escritura reduciendo de esta menera el riesgo de que se convierta en un Trastorno Específico de Aprendizaje y si esta dificultad ya se ha instalado en los niños en los primeros niveles de escolaridad el docente aportará con alternativas de solución que impidan su progreso en los próximos niveles.

La importancia de este estudio radica en que los docentes reflexionen sobre la necesidad de hacer detecciones tempranas de los síntomas disgráficos en el ejercicio profesional docente en los primeros niveles de Educación Básica, debido a que, dichas dificultades cuando no se detectan a tiempo pueden ser confundidas con pereza, desinterés, mala actitud para escribir, desgano, entre otras, impidiendo que los niños reciban una ayuda apropiada y oportuna. El maestro debe estar claro en el rol que desempeña en el proceso de enseñanza aprendizaje con sus alumnos y la importancia de su presencia en las aulas, saber interpretar sus conductas presentes y manifiestas para de esta manera detectar situaciones anómalas que le permita establecer intervenciones acertadas y a su vez permitan la adecuada adaptación escolar (Lewis, 2005).

El artículo aportará con percepciones de los profesores docentes en proceso de titulación sobre la Detección de la Disgrafia desde el ejercicio profesional docente, quienes presentan experiencia en trabajar con la niñez. Los beneficiarios serán los niños quienes al estar vinculados a las instituciones educativas recibirán una atención oportuna y adecuada ya sea por parte del docente o por parte de un profesional especializado en el área. De ahí que, es preciso preguntarse cuales serían las consecuencias de que los docentes no identifiquen a tiempo la Disgrafía en los primeros años de escolaridad. Ante lo cual, a manera de hipótesis se plantea que las dificultades de escritura se profundizarían hasta convertirse en un Trastorno Específico del Aprendizaje y conllevar a un retraso con especto a los escolares de edad similar.

\section{El lenguaje escrito y la Disgrafia}

El lenguaje escrito es la capacidad que posee el ser humano para transformar pensamientos en signos gráficos, para convertirlos en ideas conscientes, esta habilidad se la adquiere en la asignatura de lengua y literatura del currículo de Educación General Básica, con ciertos requisitos, es entonces donde se genera un 
vínculo entre la codificación lectora y escritora teniendo una adecuada correspondencia entre estas, por consiguiente, es importante la enseñanzaaprendizaje de la lengua escrita en forma correcta, siendo el maestro el principal guía del proceso, cuyo propósito es promover el desarrollo integral de los estudiantes, lo cual, implica una escritura legible, articulada, comprensible generando y transmitiendo mensajes. En tal sentido, el lenguaje escrito se convierte en una actividad organizada. (Yisel, 2021)

Las condiciones señaladas en el párrafo anterior, deben ser complementadas con habilidades motóricas, perceptuales, lingüísticas y conceptuales que avalen que la escritura sea legible, comprensible y se transmita mensajes apropiados. En otras palabras, es fundamental que el niño se expresa de manera escrita entendiendo y posibilitando el entendimiento de los demás. Cabe precisar que cuando la escritura es desordenada, presenta omisiones, adiciones, y/o trasposiciones de sílabas, letras o palabras; es decir, se evidencia una dificultad en la expresión escrita o presenta dificultades en la escritura, lo cual, se conoce como Disgrafía. El Manual Diagnóstico y Estadística de los Trastornos Mentales (DSM-5), refiere a este problema como un Trastorno Específico del Aprendizaje, (Jesús et al., 2017)

\section{La disgrafia}

Para comprender la disgrafia se parte de conceptos básicos como es la definición hecha por Ajuriaguerra, en su Manual de Psiquiatría Infantil, en el que refiere que "será disgráficos cualquier niño cuya escritura sea defectuosa, si no tiene un déficit importante intelectual o neurológico que lo justifique" (Martín et al., 2005). Cotidianamente se reportan niños en segundo tercero y cuarto año de Educación Básica que escriben muy despacio, con errores a pesar de no tener déficit intelectual que justifiquen dichos errores, sin embargo, su avance escolar va retrasándose en forma cada vez significativa y quedándose conforme avanza el nivel de escolaridad haciendo ver la diferencia entre sus pares, acumulando los parámetros más temidos para su diagnóstico clínico.

De acuerdo a Hernández, "La disgrafía es un trastorno funcional, es decir, que no está causado por una lesión cerebral o sensorial, ni por una deficiencia intelectual, y que afecta a la grafía, es decir a la forma o trazado, de las letras" (como se cito en López, 2015).Acertamos con la definición de Hernández, en la que se descarta tácitamente un daño a nivel cerebral, cognitivo, neurológico 0 intelectual convirtiéndola en una dificultad que aparece sin una causa aparente en niños con un nivel intelectual normal, pero al parecer sus escritos están llenos de errores como sustituciones $u$ omisiones de letras silabas o palabras, palabras escritas sin la separación adecuada, fragmentaciones incorrectas que hay que atenderlas

Defior, "Como disgrafía evolutiva se conocen las dificultades en el aprendizaje inicial de la escritura sin que exista una razón objetiva para ello, ya que los niños han tenido una escolarización adecuada, tienen una capacidad intelectual normal..." (como se citó en López, 2015, p.14). Como podemos ver los autores citados confluyen en que la disgrafia no refiere daño cerebral o neurológico, tampoco sensorial o déficit intelectual. por lo tanto, es una condición que sí puede ser abordad por el docente en el salón de clases y con todos los agentes que involucra el proceso de enseñanza aprendizaje.

Portellano, citado por Martín, et. al. (2005), refiere que las dificultades de escritura son una serie de alteraciones perceptivas motrices que aparecen entre cuatro y cinco años y que, si no se las atiende, desembocaran en cuadros de Disgrafia. Es aquí donde el carácter preventivo de la Disgrafía adquiere significativa importancia. Saber reconocer las características o indicadores de una escritura defectuosa requiere de la 
mirada del docente para ser atendida. Según citado en Escolar Rivas y Fernández en el (2004) un disgráficos es "aquel que posea una escritura defectuosa y poco legible, con letras mal formadas o deformadas" (p. 6). La escritura defectuosa, refiere a que un niño comete muchos errores tornando una escritura poco legible.

"La disgrafia es una condición que causa dificultad con la expresión escrita"(Vicente y Bayardo, 2018, p.121). Para los disgráficos coger el lápiz, colocarse en una postura correcta, escribir sin prensar el papel, mezclar mayúsculas y minúsculas omitir o sustituir es una batalla a la hora de escribir. Hay que indicar que cuando esto sucede la escritura se vuelve lenta y complicada, convirtiéndose en un elemento limitante en el proceso de enseñanza aprendizaje.

\section{Manifestaciones de la disgrafia}

"Los síntomas de la disgrafía caen en seis categorías: visual espacial, motora fina, procesamiento del lenguaje, deletreo/escritura a mano, gramática y organización del lenguaje" (Ríos y Gutiérrez, 2017, p.115). Las dificultades viso-espaciales nos refieren complicaciones con la discriminación de las formas de letras y también con los espacios entre ellas, dificultades para escribir encima de una línea y dentro de los màrgenes, dificultades para escribir, problemas para organizar palabras de izquierda a derecha. Mientras las dificultades de motricidad fina se relacionan con la forma de coger el lápiz, colorear, posiciones del brazo del cuerpo. Las dificultades de procesamiento tienen que ver con plasmar una idea en un papel y básicamente perderse en una idea. Para finalizar los problemas de deletreo se relacionan con no poder entender las reglas del deletreo, cometiendo errores en la escritura, mezclas mayúsculas con minúsculas, se cansa, borra mucho, etc.

\section{Evaluación de la Disgrafia}

En la evaluación de la Disgrafia, no solo debe evaluar signos gráficos como la caligrafía, y ortografía, sino también, aspectos de redacción, además de evaluar las fases de la escritura como aspectos de planificación, sintácticos, léxicos y motores, las dificultades de escritura pueden radicar en uno de ellos y es necesario evaluarlos, pero previo a ello se requiere una evaluación global (Haranburu, 2019). Evaluar la Disgrafía no solo significa evaluar letras, sílabas o palabras que estén bien escritas, sino más bien, evaluar en su totalidad procesos inmersos en la escritura que no se ven, pero que están afectados internamente y que en ellos puede radicar la Disgrafía.

\section{Rol del docente frente a la Disgrafía}

Se han analizado muchos conceptos sobre la Disgrafía y se encuentra nueva información acerca de esto que realmente sacude el rol del docente, las dificultades de la escritura afectan en gran medida a los niños ya que al adquirir la escritura los niños se transforman en entes autónomos de su propio aprendizaje, capaces de desenvolverse en la sociedad, es decir, ser exitosos. Es por ello, que la adquisición de la escritura en la educación formal significa un reto que debe ser evaluado y controlado para que se cumpla este objetivo, la escritura se constituye en una adquisición que debe proporcionar placer al niño, por lo tanto el docente debe aprender sobre la Disgrafía, valorar las dificultades presentes en los primeros años y anticiparse a que estas aparezcan, de tal manera que prevenga el fracaso escolar (Fabelo y Carmen, 2006)

Siendo así " ante determinadas dificultades que puedan surgir en algún momento con determinados niños, ha de adoptar medidas bien preventivas, de resolución" (Laury et al., 2017). El enfoque del profesor frente a las dificultades de aprendizaje se articular con la didáctica, con las propuestas de enseñanza de reeducación, de conocimiento y una serie de metodologías empleadas y también con la incapacidad del sistema curricular inepto de realizar y comprender las adaptaciones curriculares, 
sin embargo el docente debe prevenir ciertas dificultades que surgen en el aprendizaje de los niños con medidas ordinarias que parecen extraordinarias como resultado la importancia de la profesionalización en el contexto actual del docente para dar a tención oportuna en el caso en que alguno de sus alumnos presente dificultades en su aprendizaje.

Es necesario que todos los educandos encuentren en las escuelas el espacio adecuado para su desarrollo social y personal por lo tanto es reto para el docente actual en su formación es aprender sobre disgrafia y concientizarla ya que cada vez es más frecuente, para reconocer los indicadores de alerta es importante evaluar los procesos lingüísticos de la escritura y sus manifestaciones externas es decir en el papel, cuando hablamos de prevención evaluar su lenguaje y las nociones básicas implícitas para la escritura, esta valoración estaría señalando la existencia de una dificultad que se ajustaría a una intervención educativa inmediata y si lo digo así es porque requieren atención ya y no cuando se ha profundizado la dificultad o cuando ya hay un fracaso escolar o sentimiento de fracaso que se traduce en un autoconcepto académico bajo, traducido al ser aceptado o rechazado, a la hora de realizar la escritura en niveles de educación en donde los niños ya han adquirido la destreza de escribir (Protocolo para la intervención psicoeducativa de la dislexia $y / u$ otras dificultades específicas del aprendizaje, 2021)

\section{Detección de la Disgrafia por parte del docente}

El docente tiene el papel protagónico en este proceso pues él es que enseña, él es el que influye y produce cambios en los educandos, es por eso que la preparación del docente no solo viene de la adquisición de conocimientos en su formación inicial sino también en las reflexiones consientes sobre la educación y su gestión en la solución de problemas en forma dinámica, para esto es importante conseguir una cambio a nivel personal que demande del docente conocimientos, procedimientos y valores, siendo así una actuación urgente que mitigue el fracaso escolar e impulse la integración social del estudiante disgráficos (Cueto y Carrera, 2021)

Es importante que el docente realice un proceso de detección adecuado de la Disgrafía o cuando existe una dificultad de aprendizaje en la escritura sea en los primeros niveles o en otros dentro del aula de clase; de esta forma podrá reconocer las dificultades del alumno en el proceso lector, Según Acevedo citado en Laury et al., (2017) nos refiere que la observación es importante en la identificación de la Disgrafia y que hay que registrar lo observado para poder encontrar el origen de la dificultad, saber si las dificultades van cambiando o aumentando, el autor refiere que las observaciones se las debe realizar con frecuencia caso contrario su valor es limitado, recalca que el niño debe ser observado a través de un cierto tiempo.

\section{METODOLOGÍA}

Para el presente trabajo se realizó una investigación acción, Kemmis y Mctaggart (1992), citado en (Vicente \& Bayardo, 2018) manifiesta que " [...] aquella que requiere planificar, actuar, observar y reflexionar ...”. El enfoque utilizado fue mixto, en el que se mezcla el método, lenguaje, técnica en un solo estudio es decir cualitativo con las narraciones, descripciones y verbalizaciones de resultados que ofrecen sentido combinando con los datos cuantitativos, datos numéricos que se aprecian en tablas y gráficos estadísticos que nos acercan a las problemáticas investigadas obteniendo transformaciones sociales significativas (Pereira, 2011).

El sustento se lo realizó a través de una revisión bibliográfica. Se consultaron artículos, tesis doctorales de maestrías, revistas refrentes al temas lo cual fue una de 
las etapas más relevantes del artículo, ya que a través de ella se realizó un estudio del tema dando importancia y originalidad al trabajo definiendo los aquellos que son más interesantes como lo afirma Sabour ( 2017) "Para el proceso de investigación bibliográfica se debe contar con material informativo como libros, revistas de divulgación o de investigación científica, divulgación o de investigación científica, sitios Web y demás información necesaria para iniciar la búsqueda" (p.159).

El estudio se aplicó en la Universidad Tecnológica Indoamérica, en la sede de la ciudad de Ambato, se aplicó encuestas a los estudiantes de 3 paralelos de séptimo nivel de la Carrera de Ciencias de la Educación Básica, siendo característico que los estudiantes se encuentran insertados en la labor preprofesional, garantizando la presencia de al menos uno de ellos en cada una de las escuelas a nivel del país, tanto fiscales, particulares, fiscomisionales, unidocentes y pluridocententes.

Tabla 1:

Población Participante

\begin{tabular}{ccc}
\hline $\begin{array}{c}\text { Paralelos de } \\
\text { 7mo Nivel }\end{array}$ & $\begin{array}{c}\text { Total, } \\
\text { estudiantes }\end{array}$ & $\begin{array}{c}\text { Estudiantes } \\
\text { en ejercicio } \\
\text { profesional }\end{array}$ \\
\hline 01 & 69 & 42 \\
\hline 02 & 66 & 33 \\
\hline 03 & 55 & 27 \\
\hline Total & 190 & 102 \\
\hline
\end{tabular}

Nota: Información tomada de los Proyectos Formativos de $7 \mathrm{mo}$ nivel Dificultades de Aprendizaje de la Lecto-Escritura del subnivel de Educación Elemental Básica III

\section{Técnica e Instrumento}

La técnica utilizada para el presente artículo fue la encuesta y el instrumento fue el cuestionario el mismo que fue construido con 12 preguntas La encuesta se aplicó en una sola ocasión y estuvo dirigida a la obtención de información sobre conocimiento, identificación de la Disgrafía; para la validación de la encuesta se solicitó la ayuda de 2 docentes de la carrera de Educación Básica.

\section{Figura 1:}

Conocimiento de la disgrafía por los docentes

Como docente, conoce la Disgarfia?

102 respuestas

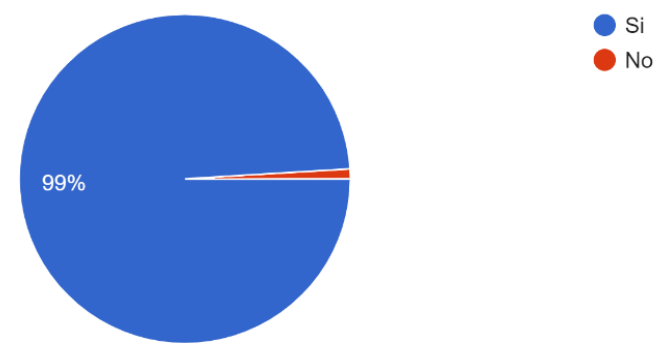

Nota. Datos obtenidos encuesta la Disgrafía. Elaboración propia 
Las respuestas evidencian que el $99 \%$ de docentes en ejercicio profesional conocen la Disgrafia.

\section{Figura 2:}

Comprensión del significado de la disgrafía por los docentes

Como docente, entiende el significado de Disgrafía?

102 respuestas

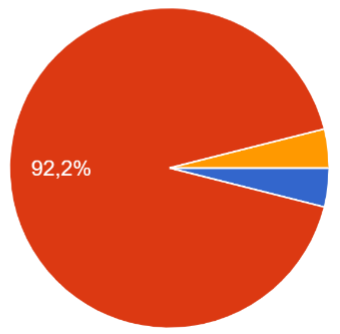

Una Dificultad Especifica de

Aprendizaje

Una Dificultad Especifica del

Aprendizaje que afecta a la escritura

Una Dificultad de Aprendizaje

Nota. Datos obtenidos encuesta la Disgrafía. Elaboración propia.

El $92,2 \%$ de docentes en ejercicio profesional contestaron que entienden a la Disgrafia como un Trastorno Específico del Aprendizaje que afecta a la escritura.

\section{Figura 3:}

Conocimiento de los docentes sobre los srrores de relacionados con la disgrafía

Como docente, ha notado que el niño disgráfico, presenta mayor cantidad de errores en

102 respuestas

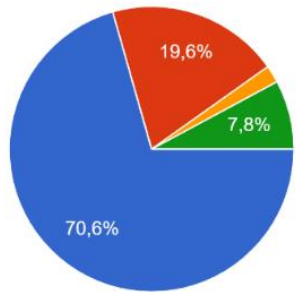

Escritura de fonemas, silabas y palabras

En la Redacción de Textos

Motricidad Fina

Escritura de frases

Nota. Datos obtenidos encuesta la Disgrafía. Elaboración propia.

\section{Figura 4:}

Deteccción de la disgrafía por parte de los docentes

Como docente, señale como detecta la Disgrafia en el Salón de Clases?

102 respuestas

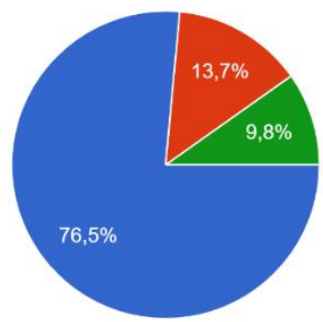

Observación en las actividade

cotidianas de la clase

Evaluación para la disgrafía

Entrevista con los padres

Evaluación de tareas

Nota. Datos obtenidos encuesta la Disgrafía. Elaboración propia. 
La encuesta permite evidenciar que un $76.5 \%$ de docentes realizan la detección de la disgrafía a través de las actividades cotidianas en el aula lo que indica que el docente ha desarrollado la observación como medio de evaluación de la escritura, además un conocimiento claro de los errores de la escritura los cuales pueden ser detectados en forma rápida, el $13,8 \%$ aplica una evaluación para la Disgrafía tal vez ven a la disgrafía como una entidad que debe ser evaluada con un instrumento específico; y ,el 9,8\% de docentes la detectan a través de la evaluación de tarea tal vez porque la evaluación de tareas implica más detenimiento, revisión, dejando sin importancia detectar la Disgrafía a través de una entrevista con los padres.

\section{Figura 5:}

Niveles educativos apropiados para la detección de la disgrafía

\footnotetext{
La Disgrafia en que niveles de Educación Básica se debe detectar? 102 respuestas
}

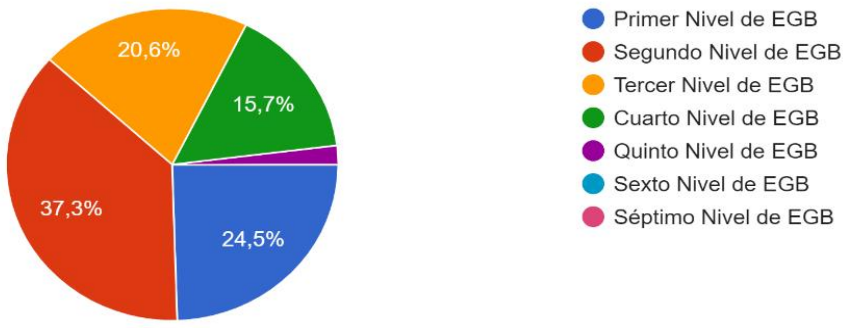

Nota. Datos obtenidos encuesta la Disgrafía. Elaboración propia.

El 37.3\% de docentes señalaron que se debe detectar la Disgrafia en Segundo año de Educación Básica es decir cuando el niño inicia su aprendizaje formal de la lectoescritura, El 24,5\% de docentes prefieren detectar la disgrafía en primer año de educación básica tal vez para prevenir que las dificultades empeoren, el $20,6 \%$ de docentes optaron por detectar la disgrafía en tercer año de Educación Básica cuando el niño recibió las habilidades de la escritura y se puede visualizar mejor los errores, el $15,7 \%$ cuando se ha consolidado la escritura y se puede ver a la Disgrafía como una entidad, restando importancia a detectar la disgrafía en niveles superiores.

\section{Figura 6:}

Progreso de la disgrafia desde la percepción docente

Como docente; ha notado que la Disgrafía cuando no se atiende 102 respuestas

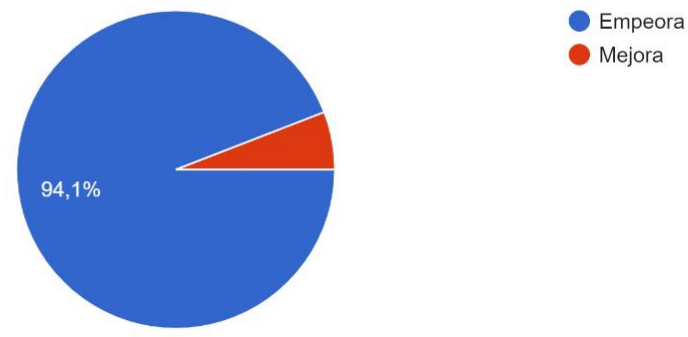

Nota. Datos obtenidos encuesta la Disgrafía. Elaboración propia. 
El 94,1 opinaron que la Disgrafia cuando no se atiende empeora y apenas el 5,9\% de docentes opinan que la Disgrafía mejora.

\title{
Figura 6:
}

Influencia de la disgrafía en el aprendizaje según los docentes
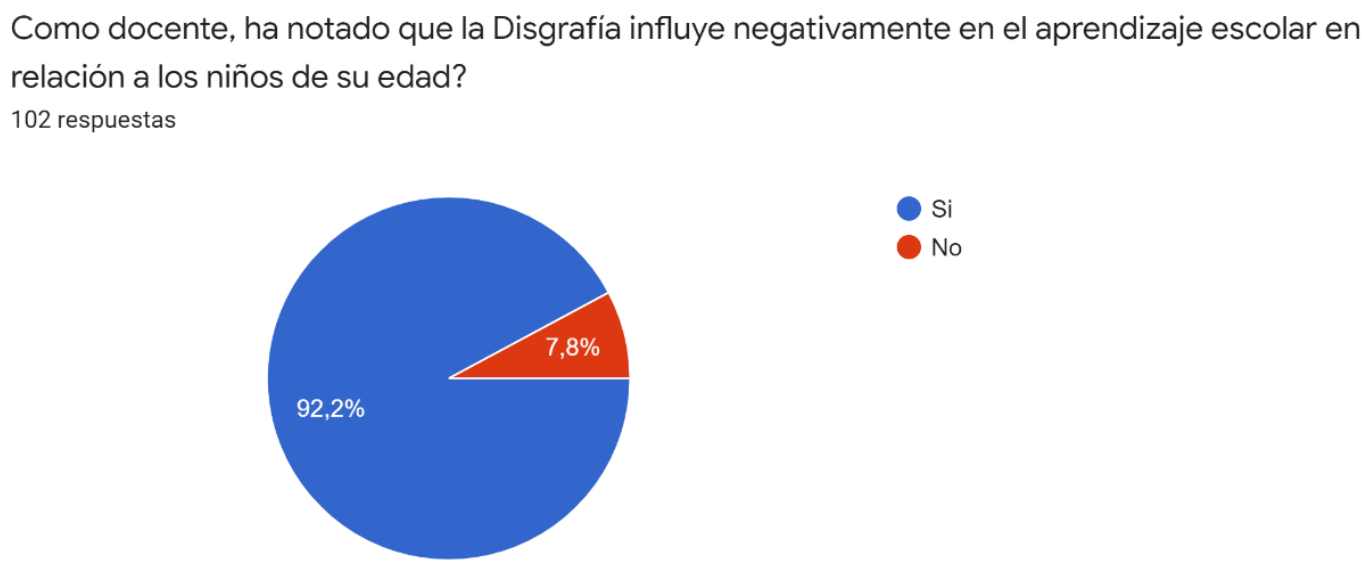

Nota. Datos obtenidos encuesta la Disgrafía. Elaboración propia.

El 92,2\% respondieron que la Disgrafía influye negativamente en el aprendizaje escolar en relación a los niños de su edad y apenas el 7,8\% respondieron que no influye en el aprendizaje.

\section{Figura 7:}

\section{Efectos de la no atención a problemas de disgrafía}

\begin{abstract}
Como docente, ha notado que sucede cuando los niños disgráficos no reciben ayuda a tiempo?
\end{abstract} 102 respuestas

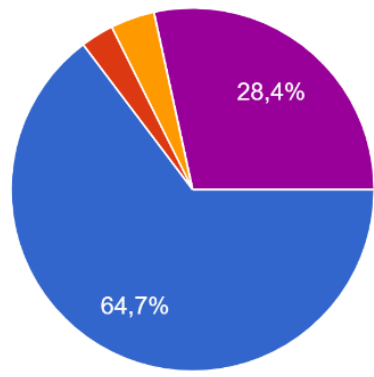

Bajo Rendimiento Escolar en relación a los niños de su edad

Repitencia del año sin haber superado la dificultad

Deserción Escolar sin completar su ciclo ni recibir ayuda

Maltrato del Docente por no aprender

Se convierte en un verdadero Trastorno de Aprendizaje

Nota. Datos obtenidos encuesta la Disgrafía. Elaboración propia.

El 64,7\% de docentes dicen los niños disgráficos al no recibir ayuda a tiempo refieren que bajan su rendimiento en relación a los niños de su edad, y el $28,4 \%$ refieren que la dificultad se convierte en un verdadero trastorno de Aprendizaje. 


\section{Figura 8:}

Niveles educativos en los que se agrava la disgrafía

Como docente, ha notado en que niveles de Educación Básica se puede agravar la Disgrafia? 102 respuestas

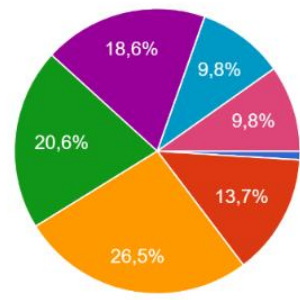

- Primer Nivel de EGB

- Segundo Nivel de EGB

- Tercer Nivel de EGB

- Cuarto Nivel de EGB

- Quinto Nivel de EGB

Sexto Nivel de EGB

Séptimo Nivel de EGB

Nota. Datos obtenidos encuesta la Disgrafía. Elaboración propia.

Un 26,5\% respondieron que la Disgrafia puede agravarse en el Tercer año de EGB, el 20,6 \% indicaron que se puede agravar en el Cuarto nivel de Educación Básica, el 18,6\% en el Quinto nivel de EGB, el 13,7\% en el Segundo Nivel de EGB, EL $9,8 \%$ en sexto nivel de EGB y el $9,8 \%$ refiere que la disgrafia se agrava en séptimo nivel de EGB.

\section{Figura 9:}

Intervención de los docentes para ayudar a estudiantes con disgrafía

Como docente, brinda su ayuda a los estudiantes que presentan problemas de disgrafia?

102 respuestas

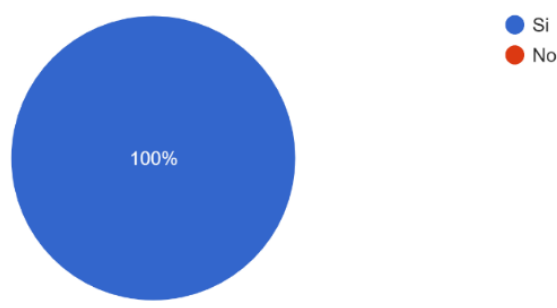

Nota. Datos obtenidos encuesta la Disgrafía. Elaboración propia.

El $100 \%$ de docentes brinda ayuda a esta dificultad

Figura 10:

Tipo de ayuda que los docentes brindan a los estudiantes con dusgrafía

Como docente, la ayuda que usted brinda a los niños con disgrafia es?

102 respuestas

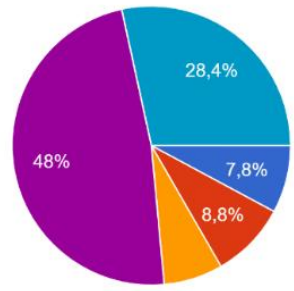

Ejercicios caligráficos en el Auta

Remite al DECE

Trabaja en el aula y con los padres de familia

Manda el refuerzo a la casa

Realiza un plan de trabajo para el niño

disgráficos

- Aplica adaptaciones curriculares

Nota. Datos obtenidos encuesta la Disgrafía. Elaboración propia. 
El $48 \%$ de docentes en ejercicio de la profesión contestaron que realizan un plan de trabajo para ayudar al niño disgráficos, el $28,4 \%$ aplica adaptaciones curriculares, el $8,8 \%$ remite al DECE y el 7,8 \% realiza ejercicios caligráficos en el aula.

\section{DISCUSIÓN}

La disgrafía es una dificultad específica del aprendizaje en la escritura que puede tener sus manifestaciones desde tempranas edades, es por ello que debe ser conocida por todos los docentes de educación para que sea reconocida por sus características y sea atendida oportunamente, es decir el docente se convierte en un representante fundamental en la prevención, los resultados nos refieren que el 100\% de docentes conocen la Disgrafia el $92 \%$ la identifican como Dificultad específica del aprendizaje que afecta a la escritura.

Las Disgrafías más comunes que se presentan son de tipo funcional, evolutivas o Primarias, en los resultados obtenidos los docentes en ejercicio de la profesión lo confirman pues un $70,6 \%$ señalan que los errores frecuentes se encuentran en la escritura de fonemas sílabas y palabras.

Los docentes son los responsables directos en la detección de esta dificultad, pues son ellos quienes observan, trabajan y evalúan a los niños por lo tanto deben estar siempre actualizados en cómo abordar la Disgrafia en el aula, saber dar recomendaciones a los padres de familia y trabajar en con ellos en planes de mejora para sus hijos para actuar a tiempo, ya sea estimulando sus habilidades 0 desarrollando estrategias para su abordaje.

Los resultados nos refieren que el 75,5 \% de docentes en ejercicio de la profesión utilizan la observación como método de detección de la Disgrafia. Los maestros deben utilizar instrumentos de evaluación apropiados para detectar la disgrafia en los niños y estar seguros de ellos pues la observación es el punto de partida que nos indica que algo no está siendo procesado de manera adecuada para el niño o que las habilidades de la escritura no se están desarrollándose de acuerdo a los niños de su edad el instrumento siempre nos ayudará a ser específicos y desarrollar estrategias adecuadas, si el caso es extremo se solicitará ayuda y orientación externa.

La formación en el docente es prioritaria, pues cada día aumentan los casos de Disgrafía en las aulas y el maestro debe estar preparado para afrontar estos retos, como ente proactivo con liderazgo en procesos pedagógicos que vengan a solucionar la problemática citada, pues las percepciones de los maestros nos indican que el 94,1 $\%$ de docentes en ejercicio refieren que la Disgrafia si no se atiende empeora, lo cual es concomitante con la siguiente percepción el $92,2 \%$ de docentes en ejercicio de la profesión responden que la Disgrafia influye negativamente en el aprendizaje escolar, por lo tanto es importante que el docente realice independientemente de las Adaptaciones Curriculares pequeños planes de intervención para el niño que presenta una disgrafía, ya que conforme pasa los niveles de Educación General básica de acuerdo a la percepción del maestro se puede agravar tal como se expresa en la pregunta en que niveles de Educación básica se puede agravar la Disgrafía a lo que se señala que de tercero a quinto nivel de mayor a menor respuestas, pues al no ser atendida esta va tomando cuerpo y convirtiéndose en un verdadero Trastorno, afectando el área afectivo emocional y conductual.

Saber específicamente cuando y en qué momento detectar la Disgrafia es clave, pero detectar los factores de riesgo de la Disgrafia es fundamental, como un factor de prevención es por ello que el docente debe formarse en conocimientos sobre este tema y reconocer aquellos factores de riesgo en los primeros niveles de educación, a 
lo que los docentes de educación en ejercicio de la profesión señalan que es importante hacer la detección en primero, segundo, tercero, cuarto nivel de EGB.

\section{REFERENCIAS}

Del, T., \& Escrito, L. (2021). Preparación de los docentes para la prevención de los trastornos del lenguaje escrito. 149-161.

Fabelo, S., \& Carmen, M. (2006). Prevención de las disgrafías escolares: necesidad de la escuela actual para la atención a la diversidad: una mirada desde Cuba. Nóesis. Revista de Ciencias Sociales y Humanidades, 15(29), 117-133.

Haranburu, M. (2019). Disgrafía: diagnóstico y tratamiento. January 2007.

Jesús, C. A., Vázquez, S., Ángeles, L. D. L., \& Fonseca, C. (2017). La dislexia, la disgrafia y la discalculia: sus consecuencias en la educación ecuatoriana. Archivo Médico de Camagüey, 21(1), 766-772.

Laury, D., GOnzález, G., \& Hernández, R. (2017). El rol del profeso ante las dificultades de aprendizaje de los niños de la primaria Álvaro Oregón de la ranchería Medellín y Madero 2da. Sección del Municipio de Centro, Tabasco. Textos y Contextos, 130, 41-49.

López, M. C. (2015). En alumnos de $3^{\circ}$ de educación primaria: la disgrafía y las pruebas de diagnóstico difficulties for the writing in pupils of 3 rd of primary education: the disgraphia and the tests of diagnosis Autor: Rebeca Pérez Fernández.

Martín, A., Barroso, J., Domínguez, M., Journal, I., \& Psychology, E. (2005). Estudio Descriptivo De La Disgrafía En Niños De $2^{\circ}$ a $4^{\circ}$ De Primaria. International Journal of Developmental and Educational Psychology, 3(1), 23-38.

Pereira, Z. (2011). Los diseños de método mixto en la investigación en educación: Una experiencia concreta. Revista Electrónica Educare, 15(1), 15-29. http://www.revistas.una.ac.cr/index.php/EDUCARE/article/view/867

Ríos, J. A., \& Gutiérrez, C. (2017). Neurobiología de los trastornos del aprendizaje y sus implicaciones en el desarrollo infantil: propuesta de una nueva perspectiva conceptual. Psicoespacios: Revista Virtual de La Institución Universitaria de Envigado, 11(19), 174-192.

Sabour, S. (2017). Reliability of smartphone-based teleradiology for evaluating thoracolumbar spine fractures: statistical issue to avoid misinterpretation. Spine Journal, 17(8), 1200. https://doi.org/10.1016/j.spinee.2017.04.017

Vicente, R., \& Bayardo, R. (2018). 687-1781-2-Pb. 3, 119-130. https://doi.org/10.23857/pc.v3i1

Yisel Santana. (2021). Estudios sobre la corrección de la disgrafía caligráfica en escolares con discapacidad intelectual Studies on the correction of calligraphic dysgraphia in schoolchildren with intellectual disabilities. Propósitos $y$ Representaciones, 9(1). 